\title{
COMMISSION 29: STELLAR SPECTRA (SPECTRES STELLAIRES)
}

\section{Report of Meetings, 19 and 25 August 1970}

PRESIDENT: M. W. Feast.

SeCRETARIES: W. P. Bidelman and L. Houziaux.

\section{Business}

The President announced with regret the deaths of two commission members: Armin J. Deutsch and Marianne C. Bretz.

Approval of the Draft Report (which few members had as yet seen) was asked and duly granted. The meeting considered that the reference problem had been satisfactorily handled in the Draft Report.

The newly-proposed Organizing Committee for the Commission was approved by acclamation.

The meeting continued with short reports by G. Cayrel on the working group concerned with equivalent-width measurement by R. Griffin on prospects for increased accuracy in this work, by C. M. Sitterly on matters connected with astronomical data centres, by G. Elste on a new compilation of results of stellar abundance analyses, and by E. Muller on the question of making available spectral Atlases and tracings of representative stellar spectra to the smaller and newer schools of astronomy.

The following resolution was proposed by W. P. Bidelman and carried with acclamation:

"Commission 29 of the International Astronomical Union, mindful of her great service to all astronomical spectroscopists, highly commends Mrs. Charlotte Moore Sitterly of the U.S. National Bureau of standards for her devoted labours in the correlation and dissemination of spectroscopic data, and expresses the earnest hope that her work in the preparation of tables of atomic energy levels and, especially, tables of the multiplet structure of the spectra of the various elements, will be vigorously prosecuted and the results made generally and inexpensively available in the future as in the past."

The following papers were read:

A. B. Severny: Measurement of Weak Magnetic Fields.

R. A. Bell: Spectrum Synthesis with a Computer.

P. S. Conti: Spectroscopic Studies of O-type Stars.

J. L. Greenstein: Helium Lines at the Hot End of the Horizontal Branch.

H. J. Wood: Balmer Line Behaviour of Magnetic and Related Stars.

Cape-Radcliffe Group (read by M. W. Feast): RY Sgr, Evidence for Pulsation and Variable Circumstellar Extinction.

T. D. Fay and A. A. Wyller: Photoelectric Observations of Stellar Line Profiles with a Pressure Scanning Spectrometer.

C. Van 't Veer: Travaux de L'équippe des Étoiles Am à Paris.

J. Hutchings: The Rotation and Extended Envelopes of Be Stars.

A. Ringuelet: Short Period Velocity Variation in $\pi$ Agr and the Group of V/R Variables.

W. P. Bidelman: Some Interesting Stars Discovered in the Michigan Southern Hemisphere Survey.

V. L. Khokhlova: Silicon Abundances in Si Ap Stars, Especially HD 124227.

J. R. Angel, J. D. Landstreet: Polarization Studies of White Dwarfs.

In addition the following joint meetings with other Commissions took place.

Joint Meeting with Commission 36: 'How to Determine Abundances'. Chairman: Greenstein.

A. B. Underhill: What Does One Need to Know to Determine Abundances?

R. F. Griffin: How Accurate are Measurements of Equivalent Widths and Line Profiles? 
B. E. J. Pagel: Uses and Limitations of the Differential Curve of Growth Method.

L. H. Aller: Use of Line Profiles in Abundance Work.

R. Cayrel: Comparison of High Dispersion Studies with Scanner Work.

A. Unsöld: General Survey of Recent Experimental and Theoretical Work on the Abundance Problem.

Joint Meeting with Commission 27: 'The Spectra of Variable Stars'

The meeting was devoted chiefly to Eta Carinae.

B. E. J. Pagel: Spectral Analysis of Eta Carinae.

A. Feinstein: The Stars Near Eta Carinae.

L. H. Aller: A Comparison of the Shell and Core Spectra of Eta Carinae and RRTel.

R. Viotti: The Excitation of the Emission Lines and Some Consideration of the Nature of Eta Carinae.

A. D. Thackeray: The Spectrum of Eta Carinae 8000-11000 $\AA$ and a Comparison of RRTel and

S. Dor with Eta Carinae.

In addition the following papers were read:

H. Maehara, Y. Fujita: Spectral Analysis of Long Period Variables.

G. H. Herbig, R. R. Zappala: The Infra-Red Spectra of NML Cygnus and the Leo Infra-Red Object IRC $+10^{\circ} 216$.

\section{WORKING GROUP ON LINE INTENSITY STANDARDS}

To increase the efficiency of this working group we suggest that somebody (e.g. Dr R. F. Griffin, if willing) should go to the McMath Solar Telescope and take photoelectric tracings with double pass of bright standard stars - (e.g. $\alpha$ Boo, $\varepsilon V i r, \alpha C M i)$.

The resulting reductions can then be used as a criterion by which to judge the accuracy of given telescope-spectrograph-observer reduction combinations at various observatories.

Suitable limited spectral regions should be investigated (the choice of which could be left to Dr Griffin). Tracings of these regions and tables of equivalent widths could then be published.

The efficiency of the subcommittee on line intensity standards will be substantially enhanced by a reduction of its membership.

We propose that the following should be members: R. F. Griffin, Koelbloed, Pagel, Wright.

Giusa Cayrel de Strobel Chairman of the Working Group 